\title{
A FAMÍLIA E OS TRANSTORNOS ALIMENTARES
}

\author{
THE FAMILY AND THE EATING DISORDERS
}

Laura Vilela e Souza'; Manoel Antônio dos Santos²

\begin{abstract}
${ }^{1}$ Psicologa. ${ }^{1}$ Mestranda em Psicologia. Bolsista da CAPES. ${ }^{2}$ Docente. Bolsista de Produtividade em Pesquisa do CNPq. Departamento de Psicologia e Educação da Faculdade de Filosofia, Ciências e Letras de Ribeirão Preto - USP.

Correspondência: Manoel Antônio dos Santos. Av. Bandeirantes, 3900, Bairro Monte Alegre. CEP 14040-901- Ribeirão Preto-SP. email: masantos@ffclrp.usp.br. Telefone: (16) 3602-3645
\end{abstract}

Souza LV, Santos MA. A família e os transtornos alimentares. Medicina (Ribeirão Preto) 39 (3): 403-9

RESUMO: Modelo do estudo: Revisão da literatura. Objetivos: Investigar as construções sobre a dinâmica familiar de pessoas com transtornos alimentares presentes na literatura especializada. Metodologia: Estudo de abordagem qualitativa com o uso da proposta teóricometodológica de produção de sentidos no cotidiano e com fundamento epistemológico na perspectiva do Construcionismo Social dentro da Psicologia Social. Foi realizada uma revisão bibliográfica utilizando a base de dados PsycINFO no período de 1972 a $2005 \mathrm{com}$ o campo de busca words anywhere com os descritores family and bulimia or eating disorders, family and anorexia or eating disorders, family and anorexia and group, family and "group therapy" and anorexia. Resultados: Foram encontradas 366 referências. Diversos estudos buscam correlações entre o funcionamento familiar e o aparecimento do transtorno, com controvérsias entre os resultados encontrados e críticas metodológicas e epistemológicas. Conclusão: Importância de uma análise crítica sobre as concepções e teorias presentes no meio científico sobre a dinâmica familiar e suas implicações nas práticas assistenciais oferecidas. Necessidade de estudos com o foco na perspectiva dos familiares sobre a doença e o tratamento.

Descritores: Transtornos da Alimentação. Bulimia Nervosa. Anorexia Nervosa. Família.

\section{1- DA FAMília do DOENTE À FAMília ADOECIDA}

Desde que a Anorexia Nervosa (AN) e a Bulimia Nervosa (BN) se tornaram objetos do campo médico-científico enquanto categorias diagnósticas específicas temos o grande desafio, enfrentado por clínicos e pesquisadores, de explicar e tratar esse fenômeno de forma eficiente. Sabemos que essas patologias são de difícil tratamento, alto risco físico e alta taxa de mortalidade ${ }^{1}$, o que aumenta a necessidade de estudos que busquem a sua compreensão. A observação de que as famílias com filhas com AN pareciam ter características de funcionamento semelhantes fez com que o contexto familiar se tornasse foco de muitos estudos que buscaram entender o papel da família no desencadeamento, manutenção e remissão do transtorno. A partir da década de 70 podemos perceber o interesse pela compreensão do fenômeno da AN por meio do modelo criado por Salvador Minuchin das "famílias psicossomáticas"2. Esse modelo preconiza que algumas características do funcionamento familiar influenciam no aparecimento de determinadas patologias. Minuchin, que era inicialmente psicanalista e mais tarde dedicou-se ao campo da terapia familiar, definiu a configuração da família psicossomática a partir das seguintes características: presença de alianças entre as gerações na família, evitação de conflitos entre todos os seus membros, repertório familiar não flexível e com pouca capacidade de resolução de problemas ${ }^{3}$. Esse modelo inspirou a concepção do que se chamou de "famílias anorexigênicas", 
ou seja, a família sendo a origem da patologia de seus filhos ${ }^{4}$. Outra terapeuta familiar, também saída do paradigma psicanalítico para adotar a abordagem sistêmica, que teve um grande interesse pelo estudo dos transtornos alimentares (TA) foi Mara Selvini Palazzoli, assim como seus colegas do grupo da escola de Terapia Familiar Sistêmica de Milão. Palazolli se impressionou com a observação dessas famílias ao perceber características muito semelhantes entre as alianças intergeracionais presentes 5 . Para a autora, a AN seria a resposta da filha (lembrando-se que no início essa era uma patologia predominante em mulheres e adolescentes) aos conflitos do processo intraconjugal de seus pais. Assim, a doença seria a resposta a padrões de interação familiar próprios dessas famílias.

Partindo da crença de que os diferentes discursos sobre esse fenômeno e sobre o papel desempenhado pela família nesse cenário, promovem práticas sociais diversas, consideramos fundamental reconhecer os diferentes enfoques adotados para o reconhecimento dessas famílias, compreendendo, dessa forma, como as intervenções propostas ao longo desses anos refletem as ideologias dos profissionais sobre a doença e suas possibilidades de recuperação. Também acreditamos ser possível, mediante essa análise, promover uma reflexão sobre as bases que fundamentam as práticas familiares atuais no contexto do tratamento, seus benefícios e limites.

Segundo o Construcionismo Social, o conhecimento e a realidade são construções sociais mediadas pela linguagem. A partir da perspectiva epistemológica do pós-modernismo que não considera a existência da dualidade sujeito-objeto, mas entende o sujeito e o objeto como construções sócio-históricas, o construcionismo convida-nos a ver a pesquisa como uma prática social. A postura ética e crítica que essa compreensão implica repercute na postura do pesquisador, que vai ser assimilado no processo da produção de sentido e que entende que não existe uma verdade única e irrefutável a ser descoberta ou um ponto de vista privilegiado para o entendimento do objeto escolhido.

A investigação construcionista preocupa-se principalmente com os processos por meio dos quais as pessoas explicam e descrevem o mundo e a si mesmas ${ }^{6}$. As crenças são geradas através dos processos de comunicação e constroem a realidade e são sustentadas pelas interações sociais. $\mathrm{O}$ projeto do construcionismo, nesse sentido, é examinar como os contextos de interação específicos privilegiam determinados discursos, compreendendo os meios pelos quais as interações são coordenadas ${ }^{7}$.

\section{2- A CONSTRUÇÃO METODOLÓGICA: BUSCANDO A FAMÍLIA DELINEADA PELA ESCRITA CIENTÍFICA}

Em nosso estudo estamos interessados em conhecer as crenças que estão por trás da doença, especialmente aquelas que destacam os fatores familiares como predição para os TA, considerando-se os valores culturais e diagnósticos vigentes. Buscamos investigar as construções sobre a dinâmica familiar de pessoas com TA presentes na literatura científica, considerando que a ciência é uma prática social e, portanto, geradora de sentidos e saber ${ }^{8}$. Realizamos uma detalhada revisão bibliográfica utilizando a base de dados PsycINFO no período de 1972 a 2005 com o campo de busca words anywhere (que permite a seleção de artigos que contenham as palavras selecionadas em "qualquer lugar" de seu texto, ou seja, no título, assunto, resumo, etc.), com os descritores family and anorexia or eating disorders, family and anorexia and group, family and "group therapy" and anorexia. PsycINFO é uma base de dados de acesso público e irrestrito da American Psychological Association, que contempla a literatura de cerca de 50 países na área da Psicologia. $\mathrm{O}$ acesso pode ser feito através da página: 〈http://www.apa.org/psycinfo>. Dessa forma pudemos ter acesso à literatura na área, que veicula os diversos discursos científicos produzidos ao longo das últimas décadas.

Para a compreensão do uso das produções discursivas científicas sobre a família nos transtornos alimentares utilizamos a análise das práticas discursivas dada pela proposta teórico-metodológica da produção de sentidos definida por Spink e Medrado9, que tem como fundamento epistemológico o Construcionismo Social na área da Psicologia Social. Essa proposta tem por objetivo a compreensão dos sentidos presentes nos diversos discursos sociais, entendendo que os conceitos e teorias presentes nesses discursos são construções dialógicas ${ }^{10}$. Os discursos são as regularidades lingüísticas que são institucionalizadas. Cada grupo social, como, por exemplo, o grupo dos cientistas, possui estruturas de poder específicas. Uma vez que os repertórios foram construídos na história e na cultura, torna-se importante retomarmos a linha da história para compreender a construção social dos conceitos encontrados na literatura científica.

\section{1- Os discursos encontrados}

Da leitura e análise dos resumos de artigos e livros encontrados foi possível depreender que diver- 
sos autores buscaram os fatores familiares específicos na predição da $\mathrm{AN}$ e $\mathrm{BN}$.

Um dos estudos mais extensos já realizados nesse campo, Strober et al. postularam as seguintes hipoteses ${ }^{11}$ :

1) $\mathrm{AN}$ e $\mathrm{BN}$ seriam familiares;

2) parentes de pessoas portadores tem maiores riscos de desenvolverem o transtorno;

3) $\mathrm{BN}$ ou $\mathrm{BN}$ parcial estaria mais presente entre familiares de pessoas com AN do que entre os sujeitos controle.

Os autores do referido estudo afirmam que os resultados oferecem novas evidências sobre o fator familiar no risco para o aparecimento do TA. Um risco 11 vezes maior para $A N$ e 3 vezes maior para $B N$. Sugerem que ambos transtornos tenham determinantes etiológicos comuns expressos nesses familiares, porém sua prevalência permanece incerta.

Em um outro estudo relevante, Bryant-Waugh e Lask estavam interessados em conhecer os fatores de risco para o aparecimento dos transtornos alimentares, salientando que, apesar do grande interesse mobilizado por esse aspecto, muito pouco se conhece sobre as causas desses transtornos ${ }^{12}$. Segundo esses autores, dentro do contexto dessa patologia existem os fatores predisponentes, que seriam o contexto no qual o transtorno aparece; e os fatores perpetuadores, que mantêm a "doença". Dentre esses últimos fatores destaca-se a desarmonia familiar, separação do casal, falta de consistência na resposta dos pais à "doença" e disfunção familiar. Os fatores familiares seriam, portanto, predisponentes para o aparecimento da patologia e não a sua causa isolada.

Macnamara e Loveman em busca de um arcabouço teórico para compreensão da dinâmica interativa disfuncional nas famílias com pacientes bulímicas ${ }^{13}$. Sobre a percepção dessas pacientes sobre a sua família, os autores afirmam que as meninas com BN percebem suas famílias como mais disfuncionais do que a percepção das pessoas das famílias controle (sem nenhum quadro patológico presente). As famílias de bulímicas apresentam pouco afeto entre seus membros, pouca comunicação e escassa habilidade para a resolução de conflitos. Esses resultados, segundo os autores, sugerem que essas jovens não aprenderam apropriadamente como controlar os seus impulsos e, dentro de uma atmosfera caótica e de pouca afetividade, buscam a comida como forma de obtenção de gratificação.
Nessa mesma linha de investigação, os resultados de um outro estudo, confirmaram a evidência de que o clima familiar seria o fator mais fortemente preditor dos $\mathrm{TA}^{14}$. Esses autores se propuseram a investigar o conteúdo do que é expresso, valorado e modelado no ambiente familiar, que pode estar fortemente relacionado ao conteúdo do sintoma que emerge no membro da família. Buscaram identificar quais são os elementos presentes especificamente no clima familiar dos pacientes com TA. Como resultados do estudo temos a observação de que nessas famílias há uma ênfase muito forte na aparência, na reputação familiar e nas conquistas pessoais, e a dieta do indivíduo refletiria a tentativa da família de se adequar aos padrões sociais de exigência de uma boa aparência e sucesso.

Berghold evidencia, em sua pesquisa, a relação entre a presença de conflito familiar e o ódio de si mesmo encontrado nos adolescentes com anorexia nervosa ${ }^{15}$.

A presença de relacionamentos maritais conflituosos levando a atitudes familiares de falsa solidariedade é um achado referido por Gensicke acerca da relação da socialização familiar com a AN, corroborando outros estudos que sustentam que uma estrutura familiar complexa fomenta condições que propiciariam uma crise adolescente, que estaria subjacente ao desencadeamento da enfermidade ${ }^{16}$.

Latzer et al. examinou a influência do ambiente familiar e dos estilos de ligação e relacionamento entre os membros nos transtornos alimentares ${ }^{17}$. Os indivíduos com esses transtornos mostraram-se menos seguros e mais ansiosos do que os indivíduos do grupo controle. Foram identificados pouco encorajamento familiar para o crescimento pessoal desses indivíduos e estilos de ligação e união instáveis.

Em um estudo realizado na China, Chan e Ma, obtiveram resultados muito semelhantes aos das pesquisas realizadas no Ocidente ${ }^{18}$; ou seja, a recusa da comida simbolizaria a disciplina aplicada ao corpo e a punição dirigida à família, como forma de vingança pelo aprisionamento vivenciado pela paciente anoréxica dentro da família. Constatou-se que a anorexia aparecia como forma de protesto e mostra de descontentamento frente à falta de autonomia da filha com relação aos pais.

As dificuldades da família de apoiar a criança durante seu processo de separação, individuação e de exploração do mundo externo também são evidenciadas por um outro estudo, conduzido por Lane, que 
aponta a existência de laços de dependência com a mãe que não encoraja suas tentativas de emancipação ${ }^{19}$. Esse estudo evidenciou, ainda, que indivíduos com transtornos alimentares provêem de lares disfuncionais, com mães controladoras e pais ausentes, além de frequientes histórias de traumas permeando os enredos familiares. Os sintomas os mantêm como crianças pequenas que rejeitam os marcos - tais como a menstruação - que evidenciam a maturidade sexual, o curso contínuo do desenvolvimento e a emergência da feminilidade no corpo em transição para a vida adulta.

Em um estudo da transmissão transgeracional da imagem corporal na família, Bonsch et al. afirmam que os aspectos negativos da experiência com o corpo parecem ter um papel importante na relação mãe e filha; ou seja, a mãe expressaria mais esses aspectos ou a filha seria mais suscetível a eles ${ }^{20}$.

Um estudo mais antigo, de Doerr et al., revelou que, na maior parte das famílias com pessoas com transtornos alimentares estudadas, pelo menos um parente com alguma doença crônica foi encontrado ${ }^{21}$. Mostrou, também, a presença de um mito familiar que não pode ser revelado, o que causa intenso estresse familiar. Outras características destacadas foram: a valorização excessiva das realizações escolares e profissionais e a rejeição da sexualidade dos filhos.

Segundo Polivy e Herman, são centrais nesses transtornos os problemas de identidade e controle pes$\mathrm{soal}^{22}$. A família contribui diretamente na exacerbação desses problemas, podendo transmitir preocupações alimentares. Porém, ainda segundo os mesmos autores, para a emergência do transtorno são necessários outros fatores de risco individuais, como déficit de auto-estima, características específicas da personalidade, experiências interpessoais, fatores estressantes da história de vida, aspectos cognitivos, influências biológicas, além de humor depressivo e irritabilidade, entre outros.

\section{3- A ANÁlise CRÍTICA dAS CONSTRU- ÇÕES SOBRE A FAMÍLIA}

O cuidado na interpretação dos resultados desses estudos é constantemente relatado. Muitos autores trazem críticas sobre as estratégias metodológicas empregadas para avaliar a correlação existente entre funcionamento familiar e o aparecimento da patologia. Algumas dessas críticas: o fato de existirem famílias que não se encaixavam nos modelos teóricos disponíveis sobre disfunção familiar ${ }^{3,4}, \mathrm{o}$ fato de a maior parte desses estudos ser de correlação e predição de resultados (desfecho do tratamento), de modo que se torna difícil determinar se a disfunção familiar contribui para o transtorno, se o transtorno contribui para a disfunção ou se simplesmente existem alguns fatores comuns a ambos ${ }^{23}$. Ademais, o papel da família é ampliado freqüentemente por perguntas retrospectivas, dificultando discernir com precisão qual foi realmente o desencadeante do sintoma.

Dentre os estudos levantados, vários sugerem a importância da inclusão da percepção dos familiares nas pesquisas, e não só dos pacientes adoecidos, o que colaboraria para uma melhor compreensão do fenômeno complexo e multifacetado da dinâmica familiar encontrada nesses transtornos ${ }^{24,25}$.

Guttman e Laporte por exemplo, mostram a diferença na percepção das relações intrafamiliares segundo mãe e filha com anorexia nervosa, sendo que a filha vê a família como menos saudável ${ }^{25}$. Corroborando esses achados, outra investigação, conduzida por Dare et al., evidenciou que os pais de indivíduos com transtornos alimentares vêem sua estrutura familiar como mais coesa e flexível do que seus filhos ${ }^{26}$. A importância da autopercepção sobre a dinâmica familiar e a causa do transtorno é enfatizada por North et al., após destacarem que a percepção objetiva da equipe de pesquisadores sobre as relações familiares foi diferente da percepção dos membros da família, que se viram como mais "normais" do que o observado ${ }^{27}$.

Em sua pesquisa, Bryant-Waugh e Lask pontuam que na sua experiência algumas famílias não chegam a apresentar qualquer distúrbio e a recuperação dos filhos ocorre após simples aconselhamento dos pais pela equipe ${ }^{12}$.

Eisler critica a base teórica da qual derivam muitas teorias sobre a dinâmica nessas famílias ${ }^{23}$. Para esse autor, a maior limitação dos estudos anteriores na área era o foco na etiologia ao invés de se buscar uma compreensão acerca de como essas famílias se organizam em torno de um problema grave como a anorexia e bulimia nervosas. Para ele, o quadro que aparece quando fazemos uma análise crítica do histórico dos transtornos alimentares, mostra que não existe um padrão consistente de estrutura familiar ou funcionamento nessas famílias. Ele afirma que o suporte empírico para a conceitualização de Minuchin é inconvincente. Não seria possível saber se o fato de termos uma evitação de conflitos nessas famílias pode ser resultante do aparecimento da própria "doença", ou se seria uma estratégia utilizada pela família para 
lidar com essa condição difícil. Ainda que se tenha evidência dessa evitação como fator de risco para o desenvolvimento do TA, ainda assim isso diz pouco sobre o porquê a anorexia apareceu naquela família. Para que pudéssemos comprovar o modelo teríamos que ter todos os componentes presentes em todas as famílias. O problema é achar que essa é uma descrição acurada da dinâmica familiar nesses transtornos, generalizando-a a ponto de que ela se torne o modelo explicatório da AN e BN. E o pesquisador conclui que o que faz esse modelo ser tão persuasivo é que de alguma maneira é oferecida uma forma de entendimento um fenômeno tão incompreensível.

Frente a uma tendência de patologização da relação mãe-filha na $\mathrm{AN}$, as terapeutas feministas criticam essa interpretação, como se a autonomia e dependência não fossem fatores que pudessem existir ao mesmo tempo. Devemos, pelo contrário, ver essa relação como potencialmente produtora de melhora no transtorno, com uma função importante dentro da família: a mãe estaria na melhor posição para facilitar a recuperação da filha, e a dependência desta com relação à mãe pode ser fonte de apoio para a filha durante o tratamento ${ }^{28}$.

Uma vez que o modelo de explicação teórica de uma dada doença leva à proposição de determinados paradigmas de tratamento, devemos considerar os riscos e as vantagens de acreditarmos que o que nós sabemos sobre a doença são metáforas possíveis de compreensão que são infinitamente relativas e negociáveis $^{29}$. Como afirma Krusky, isso não significa admitir que qualquer definição nossa sobre a família serve, ou que se justifica o abandono da responsabilidade moral sobre nossas práticas, e sim que temos que levar em conta que a nossa descrição sobre as pessoas e o mundo são construções compartilhadas sobre essa realidade, e não a verdade pronta e acabada sobre ela ${ }^{30}$.

\section{1- A inserção da família no tratamento}

A família entra na história do tratamento dos TA a partir de 1950, sendo na verdade excluída dele, pois a observação de que alguns comportamentos nessas famílias serviam para a manutenção dos sintomas dos pacientes levou os profissionais a isolarem os familiares, acreditando que a sua influência seria negativa sobre a recuperação dos adoentados.

$\mathrm{O}$ modelo de Minuchin estimulou o interesse em intervenções familiares, principalmente com a terapia familiar, que foi e ainda é muito utilizada no con- texto de tratamento dos TA. A família é envolvida, enfim, no contexto do tratamento, porém entra, muitas vezes acusada de ser "culpada" pelo transtorno de seus filhos, o que acaba gerando dificuldades no estabelecimento do vínculo com os profissionais. Para evitar que o sentimento de culpa paralizasse as famílias, algumas estratégias foram utilizadas, como comunicar à família que ela não "causa" o TA, mas que existem múltiplos fatores em conexão com o seu aparecimento. Uma outra estratégia é recomendar aos familiares que, ao invés de culparem a si mesmos, passem a culpar o problema ${ }^{31}$.

Muitos pais acham que os sintomas da filha são uma afronta direta a eles. O terapeuta vai focar quase que exclusivamente nos comportamentos bulímicos e anoréxicos, mostrando para os pais a seriedade do quadro e que suas filhas não têm o controle sobre a impulsividade de seus sintomas ${ }^{31}$.

Speed cita o exemplo de uma profissional que buscou na abordagem sistêmica tirar o foco do sintoma, buscando entender os relacionamentos e interações, bem como olhar para os sistemas culturais ${ }^{29}$. Observando que, em alguns momentos de sua prática clínica, os sintomas pareciam ficar autônomos com relação aos relacionamentos familiares e que ver a família toda junta mostrava-se em alguns casos bastante improdutivo, a autora começou a atender os pacientes também separadamente de suas famílias, em uma alternativa de atendimento que parecia mais interessante em alguns casos, mas que colocava o terapeuta familiar em uma situação de sentir-se contrário aos preceitos de sua técnica aprendida.

A partir dessas reflexões temos exemplos de modificações no relacionamento estabelecidos com esses familiares ao redor de todo o mundo. Por exemplo, na Holanda propõem-se que os membros da família estejam ativamente presentes no encaminhamento do próprio tratamento ${ }^{32}$. A equipe de profissionais observou que as diferenças e conflitos presentes no grupo e entre as equipes de internação e ambulatorial, facilitavam o aparecimento de comportamentos cindidos dos pacientes. Um dos problemas era a perda de peso após a alta; a pressão para a internação era maciça, tanto por parte dos profissionais como da família. Os pais depositavam toda a responsabilidade pelo trabalho nos profissionais da internação e essa expectativa elevada acabava levando a altas prematuras e abandono do tratamento.

Fleming afirma que essa postura é a introdução de um posicionamento mais próximo do paciente e de 
sua família ${ }^{32}$. Recomenda solicitar colaboração intensa e integral da família, visando obter a co-cooperação para a recuperação de seus filhos, o que auxilia na redução dos sentimentos de culpa. A autora e a equipe que ela representa estão convencidas da necessidade de que a família e a equipe sejam parceiras no tratamento dos TA em adolescentes.

\section{Concluindo}

Considerando os limites de nossa pesquisa, que considerou apenas os resumos dos artigos e livros publicados sobre o assunto, pudemos perceber que são poucos os trabalhos que objetivam conhecer a eficácia dos tratamentos oferecidos para os transtornos alimen- tares, assim como a experiência emocional familiar de lidar com a sobrecarga resultante desses transtornos ${ }^{33}$. Podemos perceber que dar voz aos membros dessas famílias não é apenas importante do ponto de vista da compreensão desses transtornos, como fundamental para a promoção de mudanças no paradigma de tratamento oferecido pelos profissionais de saúde.

\section{AGRADECIMENTO}

Esse trabalho foi subvencionado pela Coordenação de Aperfeiçoamento de Pessoal de Nível Superior (CAPES), mediante concessão de bolsa de mestrado à primeira autora.

Souza LV, Santos MA. The family and the eating disorders. Medicina (Ribeirão Preto) 39 (3): 403-9.

ABSTRACT: Study model: Review of literature study. Purpose of the study: To investigate the constructions about the family dynamics of people with eating disorders in the specialized literature. Method: Qualitative approach based on the "daily sense production", a theoretical and methodological proposal, well-founded in the social constructionism epistemological perspective in Social Psychology. A bibliographical search was made using the data basis PsycINFO, in the period of 1972 to 2005 with the search room: "words anywhere", with the descriptors: "family and bulimia or eating disorders", "family and anorexia or eating disorders", "family and anorexia and group", "family and "group therapy" and anorexia". Results: It was found 366 references. Several studies aim to find correlations between the familiar functioning and the appearance of the disorder, with controversial between the founded results, and methodological and epistemological criticism. Conclusion: The importance of an critical analysis of the conceptions and theories about the familiar dynamics in the scientific context, and its implications in the offered specialized care. The necessity of more studies with the focus in the familiar perspective about the disease and the treatment.

Keywords: Eating Disorders. Bulimia Nervosa. Anorexia Nervosa. Family.

\section{REFERÊNCIAS}

1 - American Psychiatric Association. Practice guideline for eating disorders. New York: American Psychiatric Association; 1993.

2 - Minuchin S, Baker L, Rosman BL, Liebman R, Millman L, Todd TC. A conceptual model of psychosomatic illness in childhood. Arch Gen Psychiatry 1975; 32: 1031-8.

3 - Dare C, Eisler I, Colahan M, Crowther C, Senior R. Asen E. The listening heart and the chi square: clinical and empirical perceptions in the family therapy of anorexia nervosa. J Fam Ther 1995; 17: 31-57.
4 - Gowers S, Bryant-Waugh R. Management of child and adolescent eating disorders: the current evidence base and future directions. J Child Psychol Psychiatry 2004; 45: 63-83.

5 - Palazzoli MS. Self starvation: from the intrapsychic to the transpersonal approach to anorexia nervosa. London: Chaucer; 1974.

6 - Gergen KJ. Realities and relationships: soundings in social construction. London: Harvard University Press; 1999.

7 - Mcnamee S. A reconstrução da identidade: A construção comum da crise. In: Mcnamee S, Gergen KJ, ed. A terapia como construção social. Trad. CO Dornelles. Porto Alegre: Artes Médicas; 1998. p. 223-38. 
8 - Mirin LYL. Garimpando sentidos em bases de dados. In: Spink MJ, org. Práticas discursivas e produção de sentidos no cotidiano: aproximações teóricas e metodológicas. São Paulo: Cortez; 2000. p.153-82.

9 - Spink MJP, Medrado VM. A pesquisa como prática discursiva In: Spink MJ, org. Práticas discursivas e produção de sentidos no cotidiano: aproximações teóricas e metodológicas. São Paulo: Cortez; 2000. p. 41-62.

10 - Spink MP, Frezza RM. Práticas discursivas e produção de sentidos: a perspectiva da psicologia social. In: Spink MJ, org. Práticas discursivas e produção de sentidos no cotidiano: aproximações teóricas e metodológicas. São Paulo: Cortez; 2000. p. 7-39.

11 - Strober M, Freeman R, Lampert C, Diamond J, Kay W. Controlled family study of anorexia nervosa and bulimia nervosa: evidence of shared liability and transmission of partial syndromes. Am J Psychiatry 2000; 157: 393-40.

12 - Bryant-Waugh R, Lask B. Eating disorders: an overview. J Fam Ther 1995; 17: 13-30.

13- Macnamara K, Loveman C. Differences in family functioning among bulimics, repeat dieters, and nondieters. J Clin Psychol 1990; 46: 518-23.

14- Laliberté M, Boland FJ, Leichner P. Family climates: Family factors specific to disturbed eating and Bulimia Nervosa. J Clin Psychol 1999; 55: 1021-40.

15 - Berghold KM. An exploration of guilt in families with anorexia nervosa. Dissertation Abstracts Int 2002; 62: 4209.

16- Gensicke P. Anorexia nervosa: a familial socialization deficit. Z Psychosom Med Psychoanal 1979; 25: 201-15.

17 - Latzer Y, Hochdorf Z, Bachar E, Canetti L. Attachment style and family functioning as discriminating factors in eating disorders. Contemp Fam Ther 2002; 24: 581-99.

18 - Chan ZCY, MA JLC. Family themes of food refusal: Disciplining the body and punishing the family. Health Care Women Int 2002; 23: 49-58.

19- Lane RC. Anorexia, masochism, self-mutilation, and autoerotism: the spider mother. Psychoanal Rev 2002; 89:101-23.

20- Bonsch C, Raml E, Seiwald M, Rathner G. Body image of anorexic girls, their mothers and sisters: a controlled study. Psychother Psychosom Med Psychol 1993; 43: 420-7.
21- Doerr Zegers O, Petrasic J, Morales E. Role of the family in the pathogenesis of anorexia nervosa. Acta Psiquiatr Psicol Am Latina 1988; 34: 33-40.

22 - Polivy J, Herman CP. Causes of eating disorders. Annu Rev Psychol 2002; 53: 187-213.

23- Eisler I. The empirical and theoretical base of family therapy and multiple family day therapy for adolescent anorexia nervosa. J Fam Therapy 2005; 27: 104-31.

24- Chatoor I, Ganiban J, Hirsch R, Borman Spurrell E, Mrazek DA. Maternal characteristics and toddler temperament in infantile anorexia. J Am Acad Child Adolesc Psychiatry 2000; 39: 743-51.

25 - Guttman HA, Laporte L. Family members'retrospective perceptions of intrafamilial relationships. Contemp Fam Therapy Int J 2002; 24: 505-21.

26 - Dare C, Le Grange D, Eisler I, Rutherford J. Redefining the psychosomatic family: family process of eating disorder families. Int J Eat Disord 1994; 16: 211-26.

27- North C, Gowers S, Byram V. Family functioning in adolescent anorexia nervosa. Br J Psychiatry 1995; 167: 673-8.

28- Ma JLC. The diagnostic and therapeutic uses of family conflicts in a Chinese context: the case of anorexia nervosa. J Fam Ther 2005; 27: 24-42.

29 - Speed B. Editorial: perspectives on eating disorders. J Fam Ther 1995; 17: i-xi.

30 - Krusky M. Women and thinness: the watch on the eve of the feast - Therapy with families experiencing troubled eating. J Syst Ther 2002; 21: 58-76.

31 - Dodge E, Matthew H, Eisler I, Dare C. Family therapy for bulimia nervosa in adolescents: an exploratory study. J Fam Therapy 1995; 17: 59-77.

32 - Fleminger S. A model for the treatment of eating disorders of adolescents in a specialized centre in The Netherlands. J Fam Ther 2005; 27: 147-57.

33 - Santonastaso P, Saccon D, Favaro A. Burden and psychiatric symptoms on key relatives of patients with eating disorders: a preliminary study. Eat Weight Disord 1997; 2: 44-8. 